OPEN ACCESS

Edited by:

Fivos Panetsos,

Complutense University of Madrid,

Spain

Reviewed by:

Carlos Avendaño,

Autonomous University of Madrid,

Spain

Francesco Tenore,

Johns Hopkins University,

United States

*Correspondence:

Joseph Thachil Francis

Joey199us@gmail.com

${ }^{\dagger}$ Deceased

Specialty section:

This article was submitted to

Neuroprosthetics,

a section of the journal

Frontiers in Neuroscience

Received: 10 November 2021

Accepted: 24 January 2022

Published: 16 February 2022

Citation:

Francis JT, Rozenboym A, von Kraus L, Xu S, Chhatbar $P$, Semework M, Hawley E and Chapin J

(2022) Similarities Between

Somatosensory Cortical Responses

Induced via Natural Touch

and Microstimulation in the Ventral

Posterior Lateral Thalamus

in Macaques.

Front. Neurosci. 16:812837.

doi: 10.3389/fnins.2022.812837

\title{
Similarities Between Somatosensory Cortical Responses Induced via Natural Touch and Microstimulation in the Ventral Posterior Lateral Thalamus in Macaques
}

Joseph Thachil Francis $1,2 *$, Anna Rozenboym ${ }^{2,3}$, Lee von Kraus ${ }^{2}$, Shaohua $\mathrm{Xu}^{2}$,
Pratik Chhatbar ${ }^{2,4}$, Mulugeta Semework ${ }^{2}$ Emerson Hawley ${ }^{2}$ and John Chapin ${ }^{2+}$

${ }^{1}$ Cullen College of Engineering, Department of Biomedical Engineering and Electrical and Computer Engineering, University of Houston, Houston, TX, United States, ${ }^{2}$ Department of Physiology and Pharmacology, State of New York Downstate Medical School, Brooklyn, NY, United States, ${ }^{3}$ Department of Biological Sciences, Kingsborough Community College-CUNY, Brooklyn, NY, United States, ${ }^{4}$ Department of Neurology, Duke University School of Medicine, Durham, NC, United States

Lost sensations, such as touch, could be restored by microstimulation (MiSt) along the sensory neural substrate. Such neuroprosthetic sensory information can be used as feedback from an invasive brain-machine interface (BMI) to control a robotic arm/hand, such that tactile and proprioceptive feedback from the sensorized robotic $\mathrm{arm} / \mathrm{hand}$ is directly given to the BMI user. Microstimulation in the human somatosensory thalamus (Vc) has been shown to produce somatosensory perceptions. However, until recently, systematic methods for using thalamic stimulation to evoke naturalistic touch perceptions were lacking. We have recently presented rigorous methods for determining a mapping between ventral posterior lateral thalamus (VPL) MiSt, and neural responses in the somatosensory cortex (S1), in a rodent model (Choi et al., 2016; Choi and Francis, 2018). Our technique minimizes the difference between S1 neural responses induced by natural sensory stimuli and those generated via VPL MiSt. Our goal is to develop systems that know what neural response a given MiSt will produce and possibly allow the development of natural "sensation." To date, our optimization has been conducted in the rodent model and simulations. Here, we present data from simple non-optimized thalamic MiSt during peri-operative experiments, where we used MiSt in the VPL of macaques, which have a somatosensory system more like humans, as compared to our previous rat work (Li et al., 2014; Choi et al., 2016). We implanted arrays of microelectrodes across the hand area of the macaque S1 cortex as well as in the VPL. Multi and single-unit recordings were used to compare cortical responses to natural touch and thalamic MiSt in the anesthetized state. Post-stimulus time histograms were highly correlated between the VPL MiSt and natural touch modalities, adding support to the use of VPL MiSt toward producing a somatosensory neuroprosthesis in humans. 


\section{INTRODUCTION}

Our overall aim in this line of work is to find a method that would allow us to use microstimulation (MiSt) or other neural stimulation modalities and emulate natural neural responses in the somatosensory cortices (S1) and other somatosensory regions as determined necessary for the perception of touch. We hypothesize that similarity of neural responses following MiSt and tactile stimulation will translate into similarity of perceptions. On the other hand, MiSt that produces "unnatural" neural patterns will not result in "natural" touch perception. Therefore, if we can determine the best locations and patterns to produce such naturalistic neural responses, we should create more natural sensations. We may need to consider the neural response in a more extensive set of structures to fine-tune this approach to achieve this goal. Here, we present our findings in a non-human primate (macaque) model. We started with responses in S1 to natural touch as our template in which to optimize our VPL MiSt-induced responses as shown in our previous rat and simulation-based studies (Brockmeier et al., 2011; Choi et al., 2012, 2016; Li et al., 2013, 2014, 2015; Choi and Francis, 2018). We note that the results presented in this paper were recorded circa 2008 and the above optimization methods had not been developed or implemented. However, we feel these results from the macaques should be shared as we move toward human implementation of these systems, where we can directly interrogate our underlying hypothesis that more naturalistic S1 responses lead to more naturalistic sensations. The rodent somatosensory system is significantly different from humans and macaques we utilized (Francis et al., 2008). Here, we used simple non-optimized VPL-MiSt in the acute macaque preparation and show that the somatotopy between VPL and S1 is generally well-maintained with VPL-MiSt, comparable to natural touch in the macaque, as in the rodent (see section "Discussion").

It has been demonstrated that neuronal activity in the motor cortex can be used to directly control computer cursors and robotic systems via a brain-machine interface (BMI) (Chapin et al., 1999; Serruya et al., 2002; Taylor et al., 2002; Hochberg et al., 2006; Ganguly and Carmena, 2009; Ajiboye et al., 2017; Degenhart et al., 2020). Recently, interest in BMIs has exploded as it has become clear that such systems are likely to restore motor function lost due to spinal cord injury (SCI), neurological disease, or amputation. Such BMIs require a closed-loop configuration that uses not only real-time neural data to move an actuator, such as a robotic arm, but also delivers sensory feedback to the user (Flesher et al., 2021). To date, this feedback has come mainly through the intact visual system of the user who is viewing their performance with the BMI. However, it is known that natural reaching and dexterous tasks require somatosensory feedback for high levels of performance and control. Therefore, somatosensory feedback from sensors on a neurally controlled prosthetic arm/hand presented directly to the user via MiSt of the neural substrate should lead to a better controlled prosthetic. This somatosensory feedback, along with visual feedback, helps control such devices and allows them to become one with the user.

The use of cortical MiSt to directly introduce information into the brain has been demonstrated with some success
(Talwar et al., 2002; Fitzsimmons et al., 2007; London et al., 2008; O’Doherty et al., 2011; Flesher et al., 2016, 2021). Several investigators have shown that macro- and microelectrode stimulation in the human somatosensory thalamus (ventral caudal nucleus Vc and nearby thalamus) can produce a variety of somatosensations, including both natural and artificial, ranging from perceptions of touch or movement to sensations of hot or cold, tingling, or the sense of pressure (Lenz et al., 1995; Davis et al., 1998, 1999; Kiss et al., 2003; Ohara et al., 2004; Chien et al., 2017). In many cases, the elicited sensation depended on the stimulus frequency and its amplitude (Patel et al., 2006). Proprioceptive and cutaneous sensory modalities were found to segregate between different regions of the thalamus as described in the literature (Sacco et al., 1987; Kaas, 2007; Francis et al., 2008). This separation should help produce separate touch and proprioceptive channels for sensory input via MiSt or other stimulation modalities.

Although human thalamic studies have been beneficial in demonstrating conscious perceptions induced by (Vc/VPL) electrical stimulation, we still lack a method for producing reliably "normal" sensations. When conducting intraoperative experiments on humans, there are several constraints, such as the amount of time one must rigorously explore the stimulation state space and ethical concerns that limit the areas from which one can sample. Much work on humans has utilized large $(>1 \mathrm{~mm})$ macroelectrodes, which are often intended for deep brain stimulation (DBS) to alleviate movement disorders such as tremor or to alleviate chronic pain. This stimulation is generally at high frequencies $(100-300 \mathrm{~Hz})$. Wu et al. have suggested that such macrostimulation may activate a neural area 4000fold greater than MiSt (Wu et al., 2001). This more extensive activation may cause tingling or other paresthetic sensations through the widespread recruitment of axons and neurons.

With these limitations in mind, as an initial step toward developing an optimized somatosensory neuroprosthesis in humans, we have utilized multielectrode neurophysiological techniques in macaques (Macaca radiata) to determine how thalamic MiSt might be used to evoke neural responses in the somatosensory cortex (S1). As the macaque has a similar somatosensory stream to humans, it is a more suitable animal model for such work as compared to the rodent model (Kaas, 2007; Francis et al., 2008). Our protocol involved implantation of multi-electrode arrays in the hand representations of the VPL (VPL; 4 electrodes) and primary somatosensory (S1) cortex (32 electrodes). These simultaneous recordings allowed us to record the response patterns of hundreds of single and multiunits in the S1 cortex during computer-controlled natural touch stimulation and VPL-MiSt in equivalent somatotopic areas. These acute experiments allowed us to directly and quantitatively compare the post-stimulus responses evoked by natural touch and VPL-MiSt in S1 in anesthetized subjects intraoperatively. We used MiSt in the low-frequency range $(\leq 5 \mathrm{~Hz})$, with the work presented here held to just one biphasic MiSt pulse in the VPL. Here, we show that simple MiSt in the VPL elicits S1 cortical responses with similar properties, such as amplitude and duration, to those induced via natural touch, at least in the anesthetized state. Thus, we add evidence that utilizing such VPL-MiSt may be suitable for a somatosensory neuroprosthesis. 


\section{MATERIALS AND METHODS}

All work adhered to NIH Guide for the Care and Use of Laboratory Animals and was approved by SUNY Downstate's IACUC and followed the recommendations of the Weatherall report, "The use of non-human primates in research." All efforts were made to minimize animal suffering, including anesthetics for all surgical procedures (see below). For this work, animals were given ad lib food and water.

\section{Surgical Preparation and Recordings}

All experiments were conducted in the acute anesthetized preparation. Two female and one male monkey (Macaca radiata) were used for this study. The monkeys were initially anesthetized with Ketamine $(15 \mathrm{mg} / \mathrm{kg})$ and intubated to allow controlled ventilation and administration of Isoflurane at $0.5-3 \%$ in $(95 \%$ $\mathrm{O}_{2}$ ). Fentanyl (I.V.) $2-5 \mathrm{mcg} / \mathrm{kg} / \mathrm{h}$ was used throughout the surgery to further ensure no pain would be felt. After anesthesia had been established, the animal was placed in a stereotactic frame. A midline incision was made, and the skin retracted above the central sulcus. Craniotomies were performed directly over the arm regions in S1 and above the VPL thalamic nucleus [see atlas (Paxinos et al., 2000)]. We first implanted the S1 cortical electrode arrays in the granular layers and then performed a series of natural touch stimulation experiments to define the precise skinto-cortex representation in that animal. The implanted cortical electrodes were left in place for the remainder of that experiment.

Next, we slowly drove the thalamic electrode array into the VPL thalamus guided by the macaque atlas (Paxinos et al., 2000). We stopped at $100 \mu \mathrm{m}$ intervals to record neuronal RFs and then MiSt at different currents while simultaneously recording multi and single-unit activity from the S1 electrode array. We obtained large data sets that provided a comprehensive record of the somatotopographic relationships between the skin, the VPL, and the S1 cortex. Subsequently, we searched a subset of the VPL stimulus parameter space. To maintain consistency of the subjects for a given experiment, the macaques hand was held in place with a ring stand and flexible cord. At the same time, the tactor was attached to a second ring stand and positioned to touch the desired region of skin. Here, tactor refers to our mechanical actuator that produces a tap on a given point of the skin. The actual contact area was approximately $1 \mathrm{~mm}$ and consisted of a thin wooden dowel.

We will refer to the brain region activated by touch of a single point on the periphery as a Stimulus Field (SF) compared to a Receptive Field (RF), which is the peripheral domain that can elicit a response from a single neuron. Specifically, the region of S1 that is activated by either touch of a point on the periphery, may be denoted as S1-SF, and for VPL-MiSt at a point in the VPL as a VPL-MiSt induced S1-SF.

\section{Electrodes}

The S1 implanted electrodes consisted of an array of 32 sharp (35 $\mu \mathrm{m}$ diameter) Tungsten electrodes for acute macaque experiments (Microprobes). The S1 array was arranged in two parallel rows, each row with 16 electrodes with an intrarow spacing of $\sim 300 \mu \mathrm{m}$ and $1 \mathrm{~mm}$ inter-row spacing. After craniotomy and removal of the dura to expose the hand area in the vicinity of the central sulcus, the electrode array was positioned on the lip of the post-central gyrus such that the anterior row of electrodes was placed about $1 \mathrm{~mm}$ caudal to the post-central sulcus lip and its parallel row was placed $1 \mathrm{~mm}$ caudal to that. This puts the electrode array in area 1 on the cortical surface but into area $3 \mathrm{~b}$ as one drives the array deeper (Paxinos et al., 2000). We have pooled our data in much of the analysis here and thus do not make claims to be recording from area 1 or $3 \mathrm{~b}$ specifically.

All the electrode tips were placed flush on the cortex and then slowly driven down until layers III-IV were reached. These electrodes then remained in place, allowing the same single and multiunit clusters to be recorded simultaneously during up to 165 stimulation experiments. We would then drive our VPL array, which consisted of four sharp stainless-steel electrodes in a horizontal square array with $1.0 \mathrm{~mm}$ separation into the VPL. This VPL array was used for both recording and stimulation. The VPL array was progressively driven down through the VPL's somatotopic representation of the cutaneous periphery, allowing us to record and stimulate for a series of experiments. For both the VPL and S1 we drove the electrodes down over the course of the set of experiments until we no longer obtained responses to peripheral stimulation. For S1 this was a maximum depth of approximately $4-5 \mathrm{~mm}$, and $\sim 2.5 \mathrm{~cm}$ for the VPL.

\section{Neural Recording and Analysis}

The Plexon Inc., multichannel acquisition processor (MAP) system was used for online data acquisition and spike discrimination. An offline sorter was used for post hoc re-discrimination. The Plexon Offline Sorter provided a variety of methods for post hoc single unit discrimination. Conventional approaches were used for general spike separation and removal of stimulus artifacts. Data analysis utilized the NEX neurophysiology analysis system and its Matlab and Excel extensions. Statistica was used for statistical analysis and plotting.

We simultaneously recorded multiple neuronal waveforms from each of the electrodes and then performed offline discrimination. First, we lumped together the neural recordings from each electrode, allowing accurate estimation of the neural population responses from each cortical location. We then used a peak detection algorithm to find the maximum response from all electrodes. The simplest and most reliable method was to record the multi-unit activity from each electrode, use computer algorithms to measure the maximal response peaks and background activity in post-stimulus histograms, and then convert the response amplitudes into Z-scores, which could be normalized across the entire electrode array. One can calculate the probability of a given z-score using the Cumulative Distribution Function for the standard normal distribution that has mean $=0$ and standard deviation $=1$, which is what the z-score distribution has.

To minimize contamination from the VPL MiSt artifact, we blanked out the first $2 \mathrm{~ms}$ following stimulation, which should be under the amount of time it takes for conduction of an action potential from the VPL to the S1 cortex as well as subsequent action potential generation in the $\mathrm{S} 1$ cortical recipient neurons. In 
addition, we sorted the stimulus artifact as a unit in our template sorting method stated above and did not include these "units" representing the stimulus artifacts, which are very stereotypical and easily clustered, in our analysis.

\section{Tactile Stimuli}

Mechanical touch stimuli were applied to different regions of the hand and forearm using a computer-driven vibromechanical actuator to deliver mechanical pulses to the skin. Our standard stimulus was a single pulse producing $<1 \mathrm{~mm}$ skin displacement for $\sim 1 \mathrm{~ms}$, delivered at rates of $5 \mathrm{~Hz}$. The touch experiments involved serially tapping up to 12 locations on the hand. These results were then compared with electrical MiSt in the VPL. A single experiment lasted for $90 \mathrm{~s}$ and included tapping at only one position on the hand. Likewise, all microstimulation experiments lasted for $90 \mathrm{~s}$ and included stimulating in one electrode configuration with a given stimulus waveform.

\section{Multichannel Microstimulator}

We developed a modular 16-channel bipolar constant-current MiSt system capable of producing any arbitrary pattern of brain stimuli through multi-electrodes. Single and/or 2-electrodes were employed to produce MiSt. All VPL stimuli were made using bipolar stimuli to minimize the stimulus artifact through closely spaced pairs of electrodes. All stimuli were biphasic, normally with the anode first as utilized in the human Vc (Patel et al., 2006). Cathode-first trials were also investigated but did not produce obvious differences. MiSt pulse widths ranged from 100 to $500 \mu \mathrm{s}$. Stimulus currents ranged from 25 to $100 \mu \mathrm{A}$. Stimuli consisted of a single biphasic pulse. For all the data presented in this paper, the MiSt was biphasic and bipolar utilizing $200 \mu \mathrm{sec}$ duration phases with approximately zero inter-phase duration and presented at $5 \mathrm{~Hz}$. We did not specifically search the MiSt state space for exact thresholds; instead, we used 25, 50, 75, and $100 \mu \mathrm{A}$ as our test amplitudes. These amplitudes were chosen after brief preliminary work that spanned responses from "weak" to "strong" and enveloped the natural touch responses, as can be seen in the figures. In addition, we chose $5 \mathrm{~Hz}$ as a compromise of being able to present enough stimuli for each location in a short period of time while allowing the brief cortical response to subside before the next stimulus.

\section{RESULTS}

A total of 357 recording experiments were conducted on three macaques. All utilized simultaneous recordings from the S1 cortical hand area using spaced electrode arrays consisting of two rows of electrodes $(2 \times 16$ electrodes $)$. These experiments yielded neural activity from stimulus fields driven by a natural touch of the hand, or MiSt in the VPL at varying stimulus intensities, where a stimulus field is defined as the brain region responsive to touch at a single point on the periphery, or MiSt at a single point in the VPL. Each experiment typically involved approximately 450 stimulus presentations (at $5 \mathrm{~Hz}$ ) of touch or VPL MiSt. All macaques also received a $2 \times 2$ electrode array implanted in the VPL thalamus.

\section{Qualitative Results}

In Figure 1, we have plotted the raw post-stimulus-rasters and their associated post-stimulus-time-histograms (PSTHs) below them induced via a natural touch of the fingers. For plots with numbers less than 8 (e.g., sig001i), these are multiunit activity recorded from the VPL, and channels above 8 (e.g., sig021i) are from the S1 cortex. Notice the variety of responses, some with early phasic response, others with a later phasic response, and some with both an early and a late response such as sig001i. In Figure 2, we have plotted the same type of activity as in Figure 1, but for the MiSt-induced responses in the cortex. In Figure 2, all the raster histogram pairs are in response to $25 \mu \mathrm{A}$ biphasic stimulation. In addition, for two multi-units, we have plotted the responses to several amplitudes of MiSt as denoted in the key. Note the different scales on the $y$-axis. Most of these units that had a significant response also had a simple phasic response, as seen in Figure 1.

\section{Somatotopy}

We found an obvious relationship (somatotopy) from the peripheral touch to the VPL induced response as expected from the literature on these and other mammals (Krubitzer and Kaas, 1992; Kaas, 2007; Francis et al., 2008). In addition, we witnessed the expected somatotopy between the VPL and primary somatosensory cortex (Kaas, 2007) and found that VPL-MiSt maintained this somatotopy. Thus, VPL-MiSt on an electrode responding to digit one touch would produce S1 responses in the digit one region of $\mathrm{S} 1$.

The results from a set of typical experiments on an anesthetized macaque are shown in Figure 3, where we have drawn a cartoon of the macaque hand color-coded according to induced neural responses found in either the VPL (Figure 3C) or S1 (Figure 3B). Panel B depicts the electrode array in S1 colorcoded based on points of the hand and their associated S1-SF, with VPL-SF shown in panel C. In panel D, we show the bipolar pairs of electrodes that were used for VPL-MiSt. Note that VPL electrodes 1 and 2 had strong responses to touch (SF) while electrodes 3 and 4 had weak responses, implying that 3 and 4 were just outside the core VPL. In support of this is the fact that all pairs lead to just one S1-SF except for the strong response pair of VPL electrodes (1 and 2), which leads to a response of both S1-SFs that are concordant with those SFs seen in the thalamus. Thus, these results imply that if we have thalamic receptive fields for each of the digits as well as those tessellating the palm, we should be able to generate $\mathrm{S} 1$ cortical responses representing any portion of the hand.

In Figure 4, we describe the topographic associations between the periphery, the VPL, and the S1 cortex. Our electrode array was situated caudal to and parallel with the central sulcus. The electrode array was made of two rows of 16 electrodes each with row one electrodes 1-16 and row two 17-32. Electrodes 1 and 17 were the most lateral electrodes while electrodes 16 and 32 where the most medial. The peak induced neural activity forms two diagonal bands in Figure 4 where we have plotted the electrode numbers from 1 to 32 in a single row. There are two bands due to the two rows of the array that are plotted as a single row in 

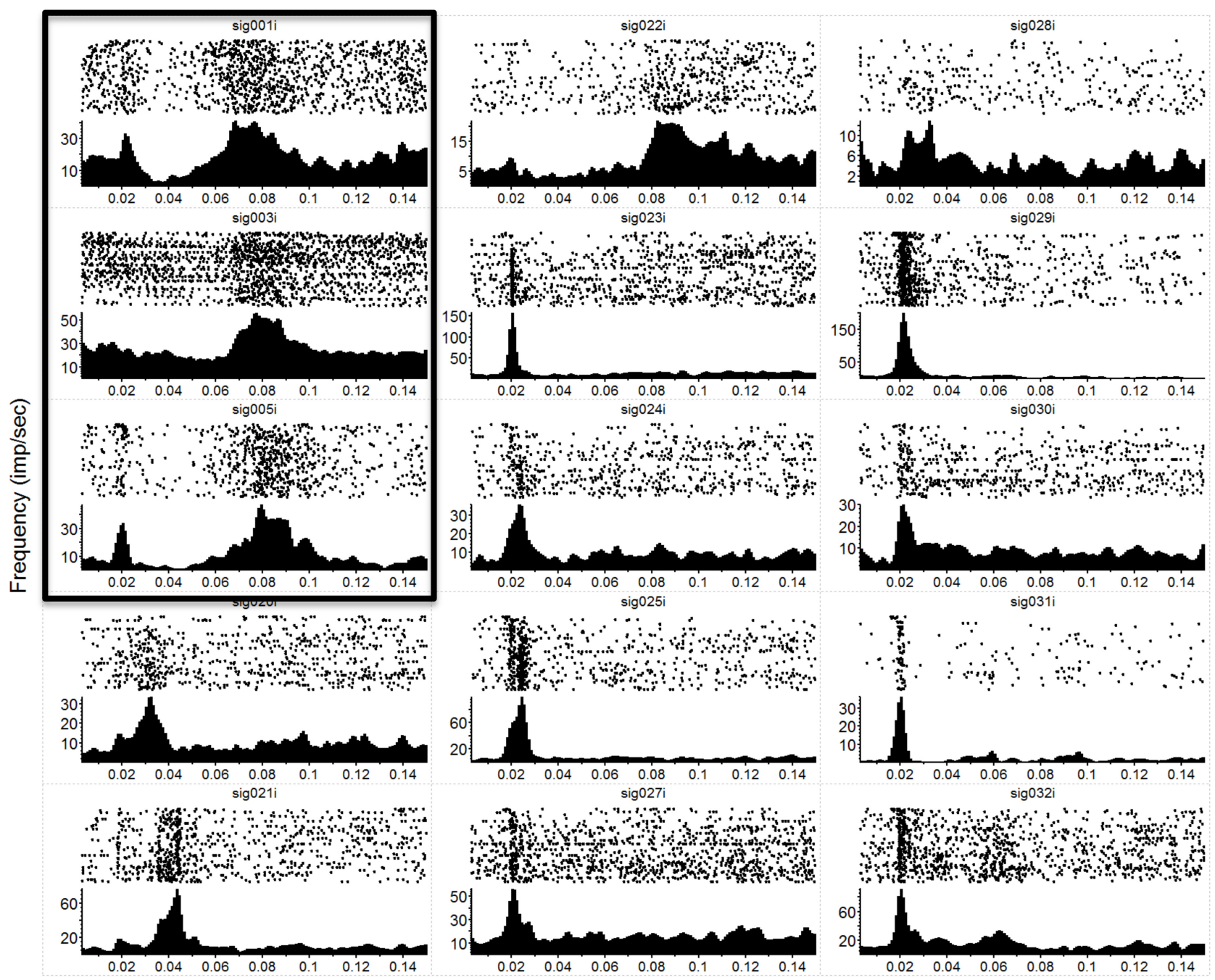

FIGURE 1 | Neural responses to mechanical stimulation. Raw data showing post-stimulus-time-rasters with their corresponding PSTHs below for a subset of the 36 recording electrodes during a single mechanical touch experiment. In this experiment, we touched the anesthetized macaque's hand at 1 position at a frequency of $5 \mathrm{~Hz}$. Each raster-histogram pair is labeled with the electrode channel number, where sig $<8$ are VPL thalamic channels (first 3 panels on the left column, marked by the surrounding box) and sig $>8$ are S1 cortical channels. The i indicates that these are unsorted units; thus, we are showing the multiunit activity recorded on each channel. There was a 4-electrode array in the thalamus $(2 \times 2$, with $1 \mathrm{~mm}$ spacing) and a 32-channel array in the cortex $(2 \times 16$, with an intra-row spacing of $300 \mu \mathrm{m}$ and inter-row distance of $1 \mathrm{~mm}$ ). Note the diversity of responses and the differences in the $y$-axis, which is the unsorted units firing rate in $\mathrm{Hz}$, while the $x$-axis is time in seconds. PSTH bins were $1 \mathrm{~ms}$ and smoothed with a 3 ms Gaussian moving window. The time axis starts at 3 ms to match the $x$-axis with Figure 2 (where MiSt stimulus artifact required blanking of the first couple of $\mathrm{ms}$ ).

Figure 4. For example, Figures 4A,B are showing electrodes 1 and 17's response to touch of D1. Note that electrodes 1 and 17 are an adjacent pair of electrodes from the two rows of the array in the rostral caudal direction. This banding simply reaffirms the known somatotopy. For instance, it is known that digit one is represented lateral to digit 4 , which can be seen in this figure as row \#1 has a peak in activity in the lateral electrodes numbered one (Figure 4A) and 17 (Figure 4B). In contrast, the peak activity for digit four is seen more medially on electrodes $3,4,5,18$, 19, and 21. As stated the color code at the right depicts the z-score for each point, e.g., $2=p<0.05$ and $3=p<0.003$. One can see in Figure 4 that some of the tactile stimulation-induced responses are focal such as for D1, whereas others are more diffuse such as for P4. As all the VPL-MiSt in these experiments were bipolar, with electrodes separated by $1 \mathrm{~mm}$, the neural responses could have multiple peaks, such as for the D4-P1,2 stimulation (Figures 4G-I), where underlining indicates this was a VPL-MiSt induced response. Figures 4C,D indicate that one row of the electrode could have a more focal response $(C)$ than the other row (D).

\section{Microstimulation Responses}

In general, MiSt at currents up to $100 \mu \mathrm{A}$ produced localized responses within S1. Figure 5A shows examples of four averaged cortical responses sequentially recorded during natural touch of Digit3 and MiSt in the VPL - Digit3 representation at 


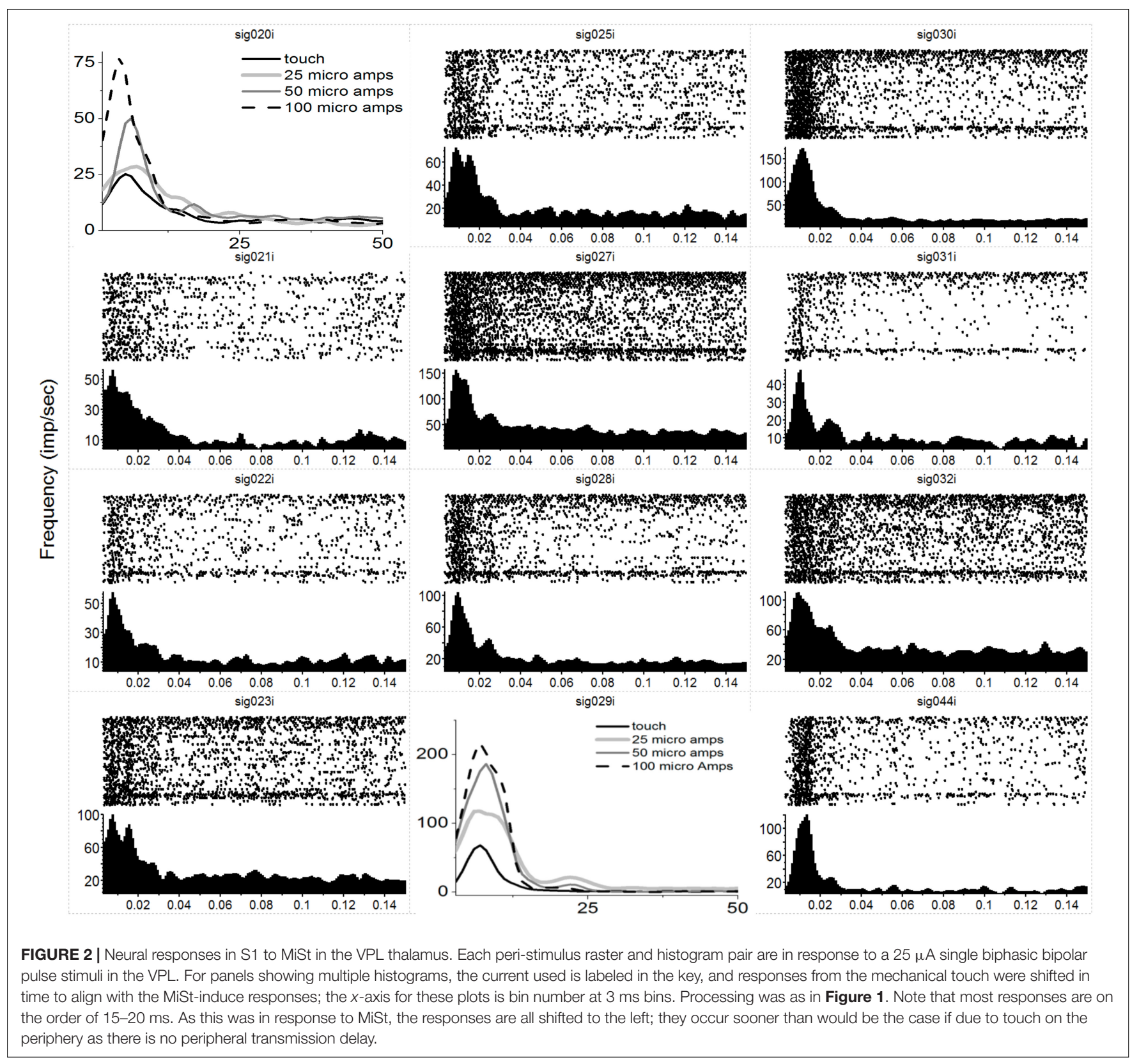

currents of 25,50 , and $100 \mu \mathrm{A}$. These results were typical in that both VPL MiSt and natural touch stimuli produced peaked cortical responses with a prominent center flanked by decaying surrounds. This basic pattern was consistent across our sample. Figure 5B shows the average of 15 cortical responses recorded during natural touch experiments and 7 from $75 \mu \mathrm{A}$ VPL MiSt. These averaged responses depict the statistical means and standard errors for each electrode in an S1 cortical array. We have aligned all the data such that the peaks correspond. These results demonstrate that: (1) VPL MiSt produces distinctly peaked cortical responses in S1. (2) The cortical response amplitudes and widths correlate with stimulus current (Figure 5A). (3) The cortical responses induced via VPL MiSt are comparable to those induced via natural touch (Figure 5B).

\section{DISCUSSION}

In this paper, we have presented results that indicate VPLMiSt in macaques can induce neural responses in S1 similar to those produced via natural touching of the hand, as we have previously demonstrated in the rat model (Choi et al., 2016; Choi and Francis, 2018). In Figure 6 we showed the $25 \mu \mathrm{A}$ VPL-MiSt S1 induced peak activity lagged the higher amplitude VPL-MiSt; further experimentation will be needed to elucidate this temporal shift. However, we expect that this difference between $25 \mu \mathrm{A}$ and the higher amplitude stimulations $(\geq 50 \mu \mathrm{A}$ ) would be due to differences in the population of neural elements exited within the high current density zone of the VPL-MiSt electrodes. It has been shown that both the density 

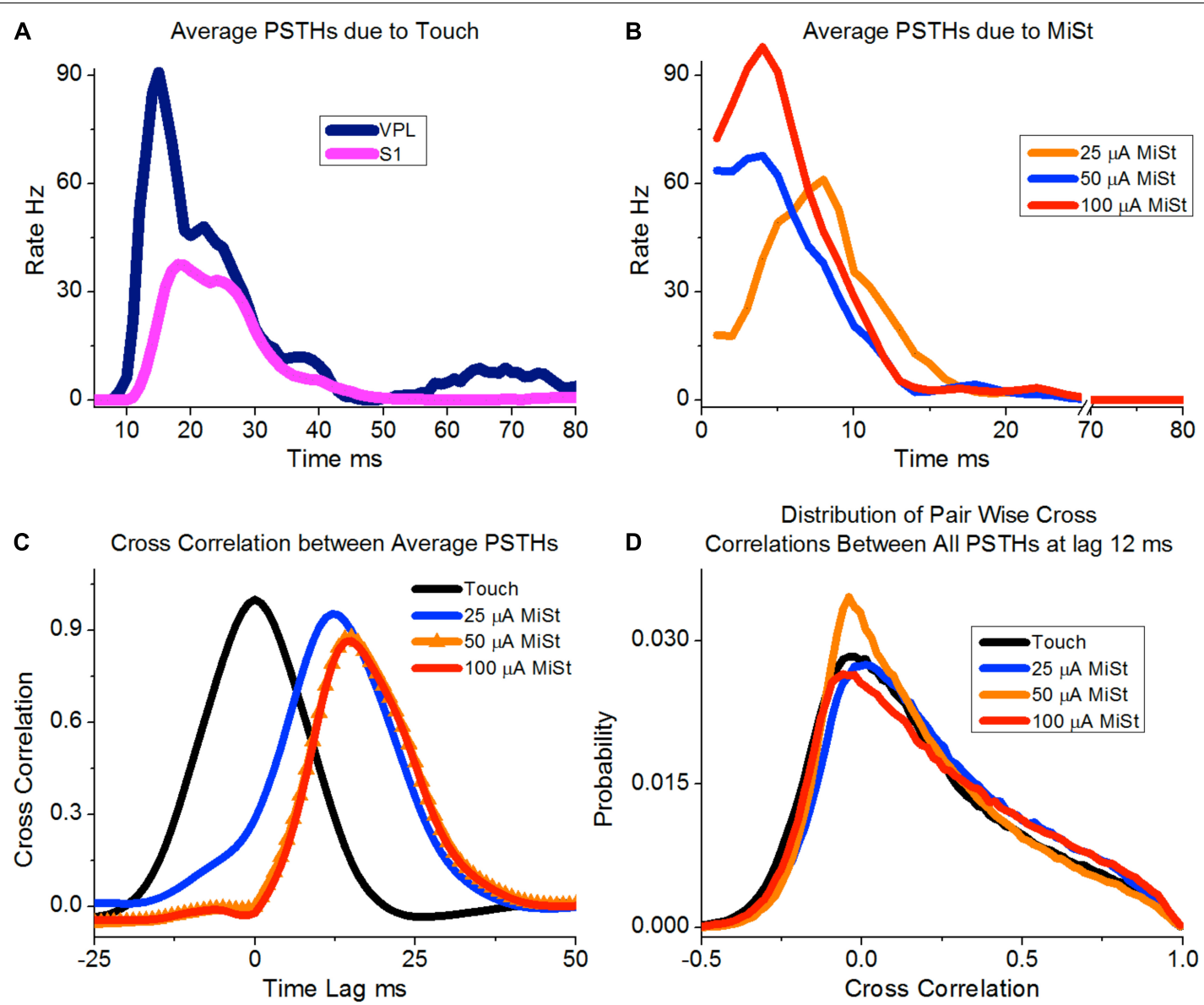

FIGURE 3 | This cartoon summarizes results showing agreement between the somatotopic relationship from VPL to S1 under natural touch (C) and VPL-MiSt (D). (A) Diagram of the macaque's hand labeled and color-coded with positions that were stimulated via our tactor. These same colors are used to describe the stimulus fields on the S1 electrodes (B) and VPL electrodes (C). (D) Are the VPL electrode pairs used for our bipolar microstimulation. Note that two of the four VPL electrodes recorded strong SF to touch $(1,2)$ while the other two were weak $(3,4)$ and possibly on the boundary of the VPL. The terms D2dors and D5dors are the digit number on the dorsal surface. Due to this arrangement, the cortical stimulus fields to VPL stimulation are governed by the strong VPL channels. See Choi et al. (2016) for a similar relationship in the rat.

of stimulated neural elements and the radius of stimulation increase with increased stimulation amplitude (Histed et al., 2009). Further work will be needed to determine how differences in such local responses propagate to other regions, such as S1, either by VPL neurons or axons going up to the cortex, antidromic activation of corticothalamic fibers and fibers going to the inhibitory thalamic Reticular Nucleus. Despite the worry that VPL stimulation might provoke widespread non-specific neural responses, we measured precise matches between the SFs in the VPL stimulus sites and the SFs in the activated regions of the S1 cortex. Small areas of single digits were readily discerned. This may be because the VPL thalamocortical fibers rapidly conduct somatotopically congruent axon bundles to circumscribed koniocortical target zones in S1. In contrast, antidromically activated corticothalamic fibers are relatively slow and dispersed. We observed that the sizes of the VPL-MiSt SFs in S1 cortex were tightly correlated with stimulus current, suggesting that the current spread approximately spherically in the thalamus before transmitting directly to the $2 \mathrm{D}$ surface of
S1. We have conducted modeling of this spread in the rat (Choi et al., 2012), which improves our ability to model VPL-MiSt and S1 activation patterns. Bipolar stimulation between two VPL electrodes spaced more than $1 \mathrm{~mm}$ apart produced two separate response areas in the $\mathrm{S} 1$ cortex. This suggests that the stimulation mainly occurred in the high-current density regions around the electrodes, which has been shown using optical techniques in other sensory areas (Histed et al., 2009).

The ability of the brief VPL-MiSt to emulate the spatiotemporal characteristics of S1 cortical responses to simple natural touch implicates the thalamocortical path as the major determinant in at least initiating these responses, as would be expected. On the other hand, direct cortical MiSt leads to widespread inhibition that typically lasts for $100 \mathrm{~ms}$ in the rat with only a very brief initial excitatory response lasting about 2 ms (Butovas and Schwarz, 2003). This could be why it generally takes trains of MiSt in the cortex for perception. Recently, it has been shown that by stimulating over several electrodes simultaneously in S1 that one can induce perception 


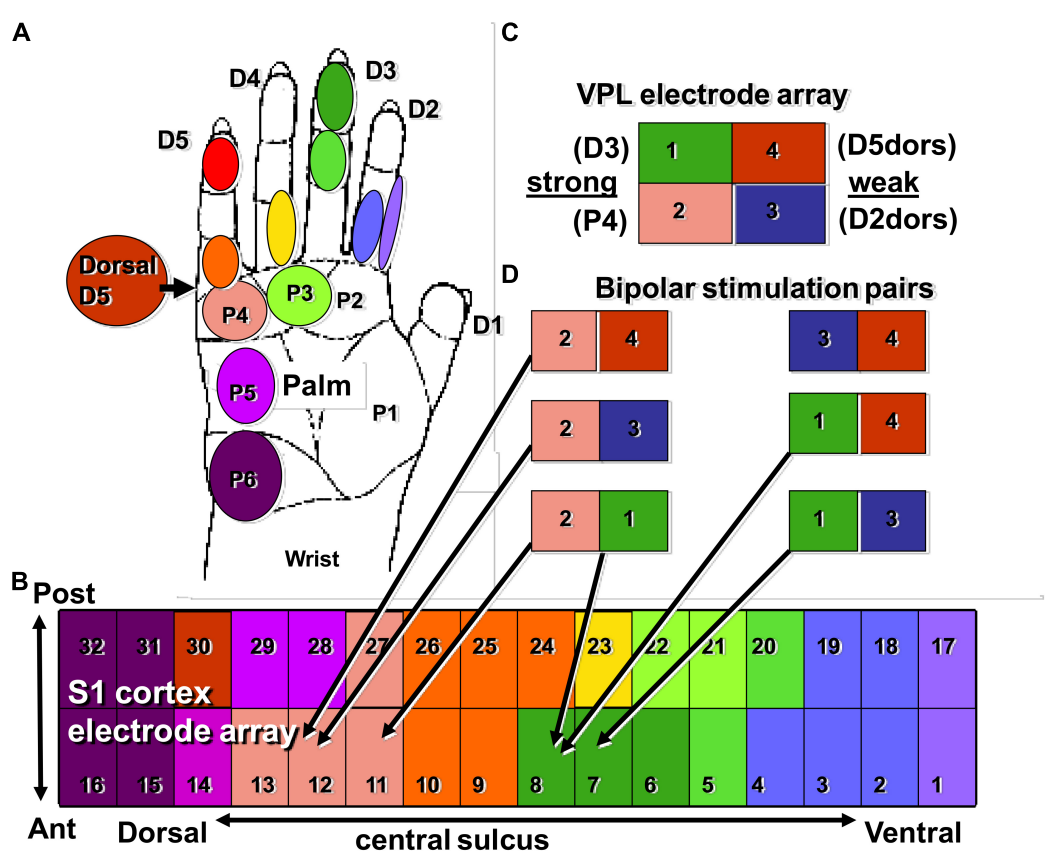

FIGURE 4 | S1 somatotopy in response to touch and VPL-MiSt. Shown are the results from 18 SFs recorded in S1 during 12 natural touch and 8 VPL-MiSt experiments (underlined, e.g., D4-Arm). VPL-MiSt was between two electrodes, where one electrode could have one SF, such as D4, and the other could be an Arm SF. Thus, the VPL-MiSt is labeled by both (D4-Arm). We have ordered the data in the expected somatotopic progression starting with D1. The color code at right depicts the z-scored significance for each point, e.g., $2=p<0.05$ and $3=p<0.003$ (see section "Materials and Methods"). See main text for reference to the points (A-I).

quicker while injecting more total current spread over the array (Sombeck and Miller, 2019). However, the authors stated the following, "RT consistently decreased when we stimulated with more current for the same number of electrodes...." "Interestingly, the RT increased as the number of electrodes increased..., an effect that was more pronounced at smaller total currents." This may not be the case for VPL-MiSt as in the current paper and our rat work, we see S1 responses like those induced by touch for single VPL-MiSt. However, this needs to be tested in the awake behaving macaque to determine if, like in humans, perceptions can be induced with brief stimuli, such as four pulses at $10 \mathrm{~Hz}$ (Patel et al., 2006). In addition, our optimized VPL-MiSt in the rat always spread the MiSt over a set of many VPL electrodes, which was likely due to our setting limits on the current the optimization could use per electrode; apparently leading to a similar conclusion reached from behavioral experiments as used by Sombeck and Miller, which indicate stimulating over several electrodes may lead to faster perception.

We found that strong VPL stimulation in macaques produced five or more oscillatory responses in the S1 cortex, while natural stimulation generally only produced 2-3 oscillatory responses. These oscillations occur at approximately $600 \mathrm{~Hz}$ and have been discussed previously (Baker et al., 2003). These results suggest that a possible mechanism for paresthesias is the highly synchronous nature of the VPL stimulation. Our conjecture, therefore, is that the ideal VPL-MiSt is one that closely approximates the response patterns produced by natural stimuli not only in the spatial extent, which has been the focus of this report but also concerning the fine temporal structure of the responses, as accomplished in our rodent work (Choi et al., 2016). However, we ultimately need to move such work into humans to address questions on the qualia of the evoked sensations.

The work we have presented was conducted with an anesthetized macaque preparation. However, we have obtained similar results in the awake restful state in rats indicating these ideas will at least hold in that neural state (Brockmeier et al., 2011). It seems prudent to replicate this in the awake macaque before moving to humans. Indeed, this is just the beginning of this work, as we expect that the awake, actively sensing state would be more complicated. We may need to consider information about other brain regions that feed into $\mathrm{S} 1$, such as motor related cortices, in addition to the current state of the S1 cortex and VPL while utilizing VPLMiSt. Recently it has been shown that S1 cortical activity is modulated by reward expectation (Pantoja et al., 2007; Ramakrishnan et al., 2017; Atique and Francis, 2021; Yao et al., 2021), punishment expectation (Yao et al., 2021) and their delivery. Indicating such affective modulation should also be tested with somatosensory neuroprostheses as this information (reward/punishment) changes neural activity within these regions and this may change perception of induced sensory neural activity.

Recently much work has been conducted using MiSt of S1 cortex directly to evoke percepts (Talwar et al., 2002; 


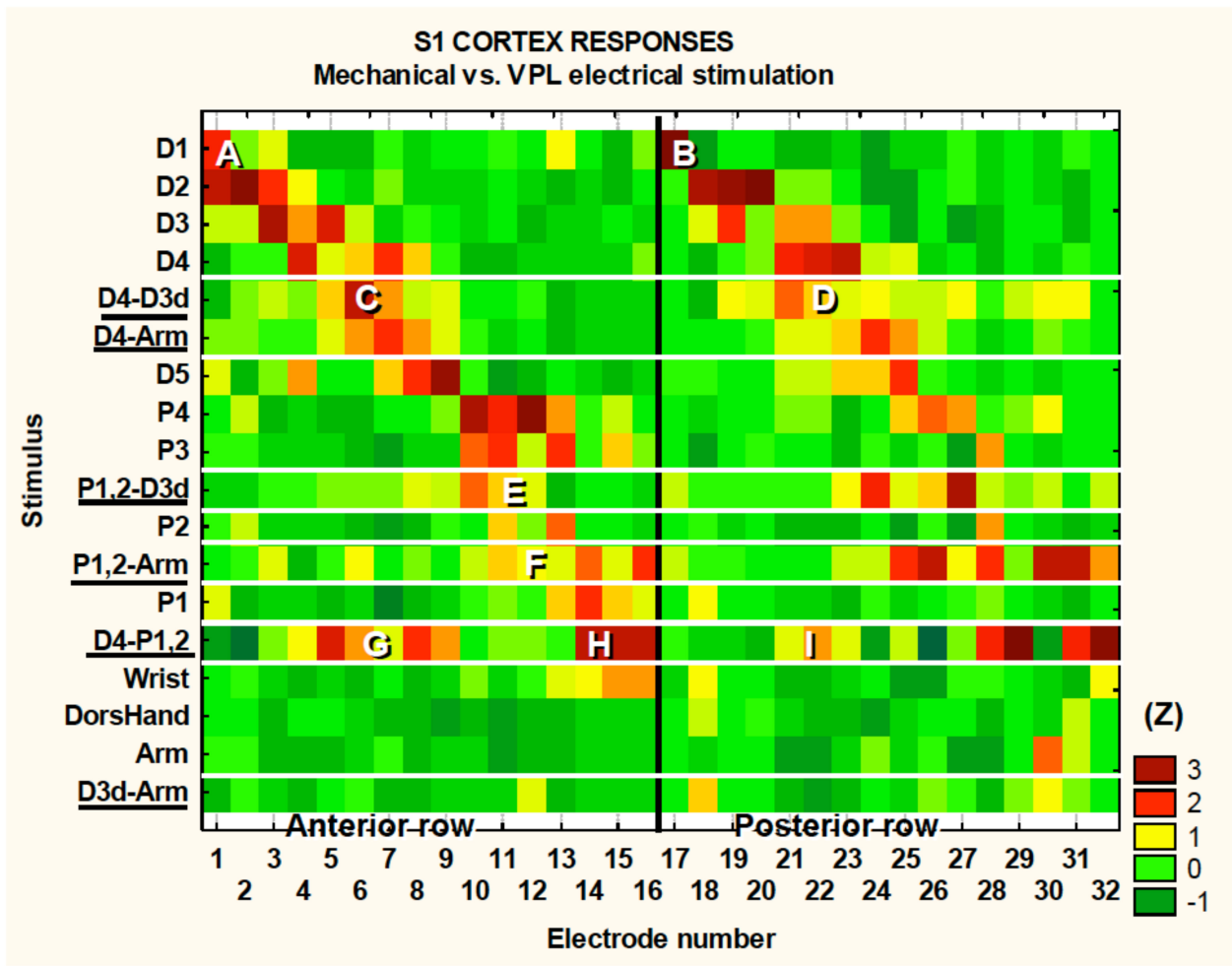

FIGURE 5 | VPL MiSt produces SFs comparable to natural touch in S1. (A) SFs produced via VPL-MiSt or touch from a single experiment. (B) Averaged stimulus fields from 15 natural touch and 7 bipolar VPL stimulation experiments. VPL-MiSt was at $75 \mu \mathrm{A}$. Each group depicts a pyramidal stimulus field \pm standard errors (error bars). Note the similarity between the two curves. All MiSt were biphasic bipolar pulses with each phase $200 \mu$ sec long. The $X$-axis is the distance in mm between cortical electrodes.
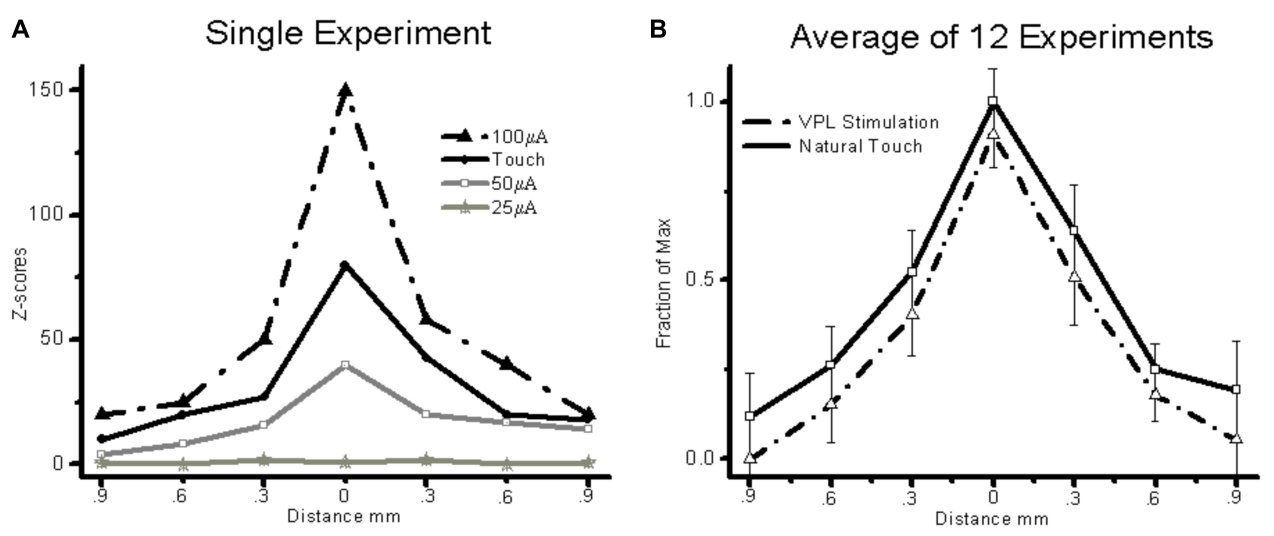

FIGURE 6 | (A) Average thalamic and S1 neural responses induced by the touch of the periphery using only channels that had a significant response for this average ( $N=1239$ ). (B) Responses for three amplitudes of microstimulation, again only using the channels with significant responses defined as peaks of 3 STDs or more ( $N=438,631$ and 420 for 25, 50, and $100 \mu \mathrm{A}$, respectively). Note that the $25 \mu \mathrm{A}$ peak occurs later than the other amplitudes. (C) Crosscorrelation between the average PSTHs for touch vs. the three MiSt amplitudes used most during our study. (D) Pair-wise crosscorrelation distribution between all pairs of PSTHs between touch and MiSt amplitudes. The touch-induced PSTHs were time-shifted by $12 \mathrm{~ms}$ as this led to the smallest sum of squared differences between the three MiSt distributions and the touch-induced distribution.

London et al., 2008; O’Doherty et al., 2011; Flesher et al., 2021). Work such as that conducted by Romo et al. (1998, 2000) has shown that S1 MiSt can be used by nonhuman primates as somatosensory information in the flutter vibration domain, while others have shown artificial proprioception via learning (Dadarlat et al., 2015). In addition, our previous work utilizing the Roborat rat paradigm has allowed us to demonstrate somatosensory neural prosthetic capabilities in a rat model. We used MiSt of the primary somatosensory cortex in this previous work with clear 
results (Talwar et al., 2002). Our preliminary results using this same paradigm with VPL-MiSt have been successful, indicating that at least in the rat, such VPL-MiSt with single biphasic stimuli, like those presented here, are perceivable by the animal (data not shown).

Our neuroprosthetic techniques utilizes the production of a neural template generated via the natural peripheral sensory organ, such as the skin for touch in our case, and working toward minimizing the difference between that template and cortical responses induced via MiSt, such as VPL-MiSt in the present case (Brockmeier et al., 2011; Li et al., 2013; Choi et al., 2016). Our model-based-methodologies (Li et al., 2013, 2015; Choi et al., 2016; Choi and Francis, 2018) work when utilizing a simulation of the periphery and neural substrate as well. This model based approach, which allows us to directly minimize S1 neurophysiological characteristics to VPL-MiSt vs. natural touch, or simulated cortical responses (Song et al., 2013; Choi et al., 2015), complements neurosurgical efforts (Hanajima et al., 2004; Ohara et al., 2004; Patel et al., 2006) that have been conducted utilizing electrical stimulation of the Vc thalamus and peripheral nerves. Significant work on Vc-MiSt has been conducted during surgical implantation of deep brain stimulators into the VIM thalamus for the treatment of tremor. Stimulation of the $\mathrm{Vc}$ in humans produces various paresthesias, especially tactile sensations in the core Vc area. Many MiSt-evoked sensations were reported as tingling, which could be related to the use of high frequency stimulation (about $150 \mathrm{~Hz}$ ). These stimulus trains were found essential for evoking somatosensory perceptions in many, but not all cases. It has been shown that just a few pulses of stimulation can induce perceptions in humans (Patel et al., 2006).

The possibility of future optogenetic implementations of the basic ideas put forth in this paper have already been shown in the retina (Nirenberg and Pandarinath, 2012). Others have described some of the difficulties with current light sensitive ion channels injected into the somatosensory thalamus (Cruikshank et al., 2010) as well as targeting these into the appropriate areas along with possible solutions to such problems (Yizhar et al., 2011). A very attractive aspect of these techniques is the fact that the VPL thalamus is a small deep brain structure that can be inject with a relatively small volume of channelrhodopsins, as compared to the cortex, and have them transported to the thalamocortical synaptic terminals (Cruikshank et al., 2010). One can then then

\section{REFERENCES}

Ajiboye, A. B., Willett, F. R., Young, D. R., Memberg, W. D., Murphy, B. A., Miller, J. P., et al. (2017). Restoration of reaching and grasping movements through brain-controlled muscle stimulation in a person with tetraplegia: a proof-ofconcept demonstration. Lancet 389, 1821-1830. doi: 10.1016/S0140-6736(17) 30601-3

Atique, M. M. U., and Francis, J. T. (2021). Mirror neurons are modulated by grip force and reward expectation in the sensorimotor cortices (S1, M1, PMd, PMv). Sci. Rep. 11:15959. doi: 10.1038/s41598-021-95536-z

Baker, S. N., Gabriel, C., and Lemon, R. N. (2003). EEG oscillations at $600 \mathrm{~Hz}$ are macroscopic markers for cortical spike bursts. J. Physiol. 550, 529-534. doi: 10.1113 /jphysiol.2003.045674

Brockmeier, A. J., Choi, J. S., DiStasio, M. M., Francis, J. T., and Príncipe, J. C. (2011). "Optimizing microstimulation using a reinforcement learning framework," in Proceedings of the 2011 Annual International Conference of the use an optical array at the larger, somatosensory cortex, at least for areas 1 and 2. However, as much of the S1 cortical region related to fine touch and proprioception is buried in the central sulcus in humans and macaques, it may still be difficult to access without causing some damage. In addition, obtaining fine spatial and temporal optical stimulation at depth below the cortical surface without causing damage is still a challenge. As high-density arrays of micro- and even nano- electrodes are evolving, it is very likely that electrical stimulation will remain the chosen intervention for clinical applications.

\section{DATA AVAILABILITY STATEMENT}

The raw data supporting the conclusions of this article will be made available by the authors, without undue reservation.

\section{ETHICS STATEMENT}

The animal study was reviewed and approved by the State University of New York Downstate Medical Center IACUC.

\section{AUTHOR CONTRIBUTIONS}

JF, AR, LK, SX, PC, MS, and JC conducted the experiments. JF and JC conducted the analysis and wrote the manuscript. $\mathrm{EH}$ produced the stimulator. All authors contributed to the article and approved the submitted version.

\section{FUNDING}

This work was supported by the DARPA Grants ONR N00149800-C-8006 and N660010-3-C-8041 JC, and N66001-10-C$2008 \mathrm{JF}$.

\section{ACKNOWLEDGMENTS}

We dedicate this work to the loving memory of JC, who passed away during the preparation of this manuscript.

IEEE Engineering in Medicine and Biology Society, Boston, MA, 1069-1072. doi: 10.1109/IEMBS.2011.6090249

Butovas, S., and Schwarz, C. (2003). Spatiotemporal effects of microstimulation in rat neocortex: a parametric study using multielectrode recordings. J. Neurophysiol. 90, 3024-3039. doi: 10.1152/jn.00245.2003

Chapin, J. K., Moxon, K. A., Markowitz, R. S., and Nicolelis, M. A. (1999). Realtime control of a robot arm using simultaneously recorded neurons in the motor cortex. Nat. Neurosci. 2, 664-670. doi: 10.1038/10223

Chien, J. H., Korzeniewska, A., Colloca, L., Campbell, C., Dougherty, P., and Lenz, F. (2017). Human thalamic somatosensory nucleus (Ventral Caudal, Vc) as a locus for stimulation by INPUTS from tactile, noxious and thermal sensors on an active prosthesis. Sensors 17:1197. doi: 10.3390/s17061197

Choi, J., and Francis, J. T. (2018). Biomimetic Multichannel Neurostimulation. US Patent 9974957. Mountain View, CA: Google.

Choi, J. S., Brockmeier, A. J., McNiel, D. B., Kraus, L. M., Principe, J. C., and Francis, J. T. (2016). Eliciting naturalistic cortical responses with a sensory prosthesis via 
optimized microstimulation. J. Neural Eng. 13:056007. doi: 10.1088/1741-2560/ $13 / 5 / 056007$

Choi, J. S., DiStasio, M. M., Brockmeier, A. J., and Francis, J. T. (2012). An electric field model for prediction of somatosensory (S1) cortical field potentials induced by ventral posterior lateral (VPL) thalamic microstimulation. IEEE Trans. Neural Syst. Rehabil. Eng. 20, 161-169. doi: 10.1109/TNSRE.2011. 2181417

Choi, J. S., Menzies, R. J., Dura-Bernal, S., Francis, J. T., Lytton, W. W., and Kerr, C. C. (2015). Spiking network modeling of neuronal dynamics in individual rats. BMC Neurosci. 16:122. doi: 10.1186/1471-2202-16-S1-P122

Cruikshank, S. J., Urabe, H., Nurmikko, A. V., and Connors, B. W. (2010). Pathway-specific feedforward circuits between thalamus and neocortex revealed by selective optical stimulation of axons. Neuron 65, 230-245. doi: 10.1016/j.neuron.2009.12.025

Dadarlat, M. C., O’Doherty, J. E., and Sabes, P. N. (2015). A learning-based approach to artificial sensory feedback leads to optimal integration. Nat. Neurosci. 18, 138-144. doi: 10.1038/nn.3883

Davis, K. D., Kiss, Z. H. T., Luo, L., Tasker, R. R., Lozano, A. M., and Dostrovsky, J. O. (1998). Phantom sensations generated by thalamic microstimulation. Nature 391, 385-387. doi: 10.1038/34905

Davis, K. D., Lozano, A. M., Manduch, M., Tasker, R. R., Kiss, Z. H. T., and Dostrovsky, J. O. (1999). Thalamic relay site for cold perception in humans. J. Neurophysiol. 81, 1970-1973. doi: 10.1152/jn.1999.81.4.1970

Degenhart, A. D., Bishop, W. E., Oby, E. R., Tyler-Kabara, E. C., Chase, S. M., Batista, A. P., et al. (2020). Stabilization of a brain-computer interface via the alignment of low-dimensional spaces of neural activity. Nat. Biomed. Eng. 4, 672-685. doi: 10.1038/s41551-020-0542-9

Fitzsimmons, N. A., Drake, W., Hanson, T. L., Lebedev, M. A., and Nicolelis, M. A. L. (2007). Primate reaching cued by multichannel spatiotemporal cortical microstimulation. J. Neurosci. 27, 5593-5602. doi: 10.1523/JNEUROSCI.529706.2007

Flesher, S. N., Collinger, J. L., Foldes, S. T., Weiss, J. M., Downey, J. E., Tyler-Kabara, E. C., et al. (2016). Intracortical microstimulation of human somatosensory cortex. Sci. Transl. Med. 8:361ra141. doi: 10.1126/scitranslmed.aaf8083

Flesher, S. N., Downey, J. E., Weiss, J. M., Hughes, C. L., Herrera, A. J., TylerKabara, E. C., et al. (2021). A brain-computer interface that evokes tactile sensations improves robotic arm control. Science 372, 831-836. doi: 10.1126/ science.abd 0380

Francis, J. T., Xu, S., and Chapin, J. K. (2008). Proprioceptive and cutaneous representations in the rat ventral posterolateral thalamus. J. Neurophysiol. 99, 2291-2304. doi: 10.1152/jn.01206.2007

Ganguly, K., and Carmena, J. M. (2009). Emergence of a stable cortical map for neuroprosthetic control. PLoS Biol. 7:e1000153. doi: 10.1371/journal.pbio. 1000153

Hanajima, R., Chen, R., Ashby, P., Lozano, A. M., Hutchison, W. D., Davis, K. D., et al. (2004). Very fast oscillations evoked by median nerve stimulation in the human thalamus and subthalamic nucleus. J. Neurophysiol. 92, 3171-3182. doi: $10.1152 / \mathrm{jn} .00363 .2004$

Histed, M. H., Bonin, V., and Reid, R. C. (2009). Direct activation of sparse, distributed populations of cortical neurons by electrical microstimulation. Neuron 63, 508-522. doi: 10.1016/j.neuron.2009.07.016

Hochberg, L. R., Serruya, M. D., Friehs, G. M., Mukand, J. A., Saleh, M., Caplan, A. H., et al. (2006). Neuronal ensemble control of prosthetic devices by a human with tetraplegia. Nature 442, 164-171. doi: 10.1038/nature04970

Kaas, J. H. (2007). "3.35 - The evolution of the dorsal thalamus in mammals," in Evolution of Nervous Systems, ed. J. H. Kaas (Oxford: Academic Press), 499-516. doi: 10.1016/B0-12-370878-8/00089-6

Kiss, Z. H. T., Davis, K. D., Tasker, R. R., Lozano, A. M., Hu, B., and Dostrovsky, J. O. (2003). Kinaesthetic neurons in thalamus of humans with and without tremor. Exp. Brain Res. 150, 85-94. doi: 10.1007/s00221-003-1399-3

Krubitzer, L. A., and Kaas, J. H. (1992). The somatosensory thalamus of monkeys: cortical connections and a redefinition of nuclei in marmosets. J. Comp. Neurol. 319, 123-140. doi: 10.1002/cne.903190111

Lenz, F. A., Gracely, R. H., Romanoski, A. J., Hope, E. J., Rowland, L. H., and Dougherty, P. M. (1995). Stimulation in the human somatosensory thalamus can reproduce both the affective and sensory dimensions of previously experienced pain. Nat. Med. 1, 910-913. doi: 10.1038/nm0995-910
Li, K., Dura-Bernal, S., Francis, J. T., Lytton, W., and Principe, J. C. (2015). "Repairing lesions via kernel adaptive inverse control in a biomimetic model of sensorimotor cortex," in Proceedings of the IEEE 7th International EMBS Conference, Montpellier. doi: 10.1109/NER.2015.7146663

Li, L., Brockmeier, A. J., Choi, J. S., Francis, J. T., Sanchez, J. C., and Principe, J. C. (2014). A tensor-product-kernel framework for multiscale neural activity decoding and control. Comput. Intell. Neurosci. 2014:870160. doi: 10.1155/ 2014/870160

Li, L., Park, I. M., Brockmeier, A., Chen, B., Seth, S., Francis, J. T., et al. (2013). Adaptive inverse control of neural spatiotemporal spike patterns with a reproducing kernel Hilbert space (RKHS) framework. IEEE Trans. Neural Syst. Rehabil. Eng. 21, 532-543. doi: 10.1109/TNSRE.2012.2200300

London, B. M., Jordan, L. R., Jackson, C. R., and Miller, L. E. (2008). Electrical stimulation of the proprioceptive cortex (Area 3a) used to instruct a behaving monkey. IEEE Trans. Neural Syst. Rehabil. Eng. 16, 32-36. doi: 10.1109/TNSRE. 2007.907544

Nirenberg, S., and Pandarinath, C. (2012). Retinal prosthetic strategy with the capacity to restore normal vision. Proc. Natl. Acad. Sci. U.S.A. 109, $15012-$ 15017. doi: 10.1073/pnas.1207035109

O’Doherty, J. E., Lebedev, M. A., Ifft, P. J., Zhuang, K. Z., Shokur, S., Bleuler, H., et al. (2011). Active tactile exploration using a brain-machine-brain interface. Nature 479, 228-231. doi: 10.1038/nature10489

Ohara, S., Weiss, N., and Lenz, F. A. (2004). Microstimulation in the region of the human thalamic principal somatic sensory nucleus evokes sensations like those of mechanical stimulation and movement. J. Neurophysiol. 91, 736-745. doi: 10.1152/jn.00648.2003

Pantoja, J., Ribeiro, S., Wiest, M., Soares, E., Gervasoni, D., Lemos, N. A., et al. (2007). Neuronal activity in the primary somatosensory thalamocortical loop is modulated by reward contingency during tactile discrimination. J. Neurosci. 27, 10608-10620. doi: 10.1523/JNEUROSCI.5279-06.2007

Patel, S., Ohara, S., Dougherty, P. M., Gracely, R. H., and Lenz, F. A. (2006). Psychophysical elements of place and modality specificity in the thalamic somatic sensory nucleus (Ventral Caudal, Vc) of awake humans. J. Neurophysiol. 95, 646-659. doi: 10.1152/jn.00756.2005

Paxinos, G., Huang, X.-F., and Toga, A. (2000). The Rhesus Monkey Brain in Stereotaxic Coordinates. Faculty of Health and Behavioural Sciences -Papers (Archive). San Diego, CA: Academic Press.

Ramakrishnan, A., Byun, Y. W., Rand, K., Pedersen, C. E., Lebedev, M. A., and Nicolelis, M. A. L. (2017). Cortical neurons multiplex reward-related signals along with sensory and motor information. Proc. Natl. Acad. Sci. U.S.A. 114, E4841-E4850. doi: 10.1073/pnas.1703668114

Romo, R., Hernández, A., Zainos, A., Brody, C. D., and Lemus, L. (2000). Sensing without touching: psychophysical performance based on cortical microstimulation. Neuron 26, 273-278. doi: 10.1016/S08966273(00)81156-3

Romo, R., Hernández, A., Zainos, A., and Salinas, E. (1998). Somatosensory discrimination based on cortical microstimulation. Nature 392, 387-390. doi: $10.1038 / 32891$

Sacco, R. L., Bello, J. A., Traub, R., and Brust, J. C. (1987). Selective proprioceptive loss from a thalamic lacunar stroke. Stroke 18, 1160-1163. doi: 10.1161/01.STR. 18.6.1160

Serruya, M. D., Hatsopoulos, N. G., Paninski, L., Fellows, M. R., and Donoghue, J. P. (2002). Instant neural control of a movement signal. Nature 416, 141-142. doi: $10.1038 / 416141 \mathrm{a}$

Sombeck, J. T., and Miller, L. E. (2019). Short reaction times in response to multi-electrode intracortical microstimulation may provide a basis for rapid movement-related feedback. J. Neural Eng. 17:016013. doi: 10.1088/1741-2552/ $\mathrm{ab} 5 \mathrm{cf} 3$

Song, W. G., Kerr, C. C., Lytton, W. W., and Francis, J. T. (2013). Cortical plasticity induced by spike-triggered microstimulation in primate somatosensory cortex. PLoS One 8:e57453. doi: 10.1371/journal.pone.0057453

Talwar, S. K., Xu, S., Hawley, E. S., Weiss, S. A., Moxon, K. A., and Chapin, J. K. (2002). Rat navigation guided by remote control. Nature 417, 37-38. doi: $10.1038 / 417037 \mathrm{a}$

Taylor, D. M., Tillery, S. I., and Schwartz, A. B. (2002). Direct cortical control of 3D neuroprosthetic devices. Science 296, 1829-1832. doi: 10.1126/science.10 70291 
Wu, Y. R., Levy, R., Ashby, P., Tasker, R. R., and Dostrovsky, J. O. (2001). Does stimulation of the GPi control dyskinesia by activating inhibitory axons? Mov. Disord. 16, 208-216. doi: 10.1002/mds.1046

Yao, Z., Hessburg, J. P., and Francis, J. T. (2021). Normalization by valence and motivational intensity in the sensorimotor cortices (PMd, M1, and S1). Sci. Rep. 11:24221. doi: 10.1038/s41598-021-03200-3

Yizhar, O., Fenno, L. E., Davidson, T. J., Mogri, M., and Deisseroth, K. (2011). Optogenetics in neural systems. Neuron 71, 9-34. doi: 10.1016/j.neuron.2011. 06.004

Conflict of Interest: The authors declare that the research was conducted in the absence of any commercial or financial relationships that could be construed as a potential conflict of interest.
Publisher's Note: All claims expressed in this article are solely those of the authors and do not necessarily represent those of their affiliated organizations, or those of the publisher, the editors and the reviewers. Any product that may be evaluated in this article, or claim that may be made by its manufacturer, is not guaranteed or endorsed by the publisher.

Copyright $\odot 2022$ Francis, Rozenboym, von Kraus, Xu, Chhatbar, Semework, Hawley and Chapin. This is an open-access article distributed under the terms of the Creative Commons Attribution License (CC BY). The use, distribution or reproduction in other forums is permitted, provided the original author(s) and the copyright owner(s) are credited and that the original publication in this journal is cited, in accordance with accepted academic practice. No use, distribution or reproduction is permitted which does not comply with these terms. 